\title{
Bridging the Social Media Usage Gap from Old to New: An Elderly Media Interpersonal and Social Research in Taiwan
}

\author{
Shih-Hsun Lin and Wen Huei Chou \\ National Yunlin University of Science and Technology \\ Department of Digital Media Design and Graduate School of Computational Design \\ 123 University Road, Section 3, 64002 Douliou, Yunlin, Taiwan \\ \{Furanke, cristance\} @gmail.com
}

\begin{abstract}
Understanding the media usage and interpersonal communication that the elderly have been familiar with is valuable for designing social media for the elderly. We conducted interviews for acquiring the data about attitudes and behaviors of the elderly, and then analyzed the transcripts to discover the patterns of the elderly in media usage and social life. The findings show that in media usage our subjects prefer watching TV and contacting people with phone/mobile phone in their leisure time. Also they prefer the habitually daily routine of watching TV in the living room although they appreciate the flexible selectivity of using a computer, but have difficulties with these new media. Activities such as weddings and funerals remain the important chances to retrieve relatives, and physical contact still remains the primary interaction for elders. Fitting in with the elderly habits in daily life to design is discussed.
\end{abstract}

Keywords: elderly, social media, media usage, media communication, interpersonal communication.

\section{Introduction}

Social media is becoming a more and more popular and influential medium of communication, especially among the younger generation. However, the elderly are accustomed to their familiar media usage to get information and communication devices to contact people, which have formed the way of the elderly media usage and interpersonal interactions in their social life. For encouraging the elderly to more easily participate in social issues, express opinions through social media, and then having their opinions to be respected, a social media that can fit into the lifestyle of those elderly is significant and crucial.

In this paper, we regarded social media as the media through which people can be connected and share information; with these uses, they can maintain relationships with friends and relatives, or even to build new relationships; those social media include social networks sites (SNS), the services implementing information and communication technologies (ICT), and computer-mediated communication (CMC) applications. 
The reformation and evolution in communications and media through information technologies and the Internet has been developing into the information society, which influences the structure of society. Technologies allow information to be distributed more easily and faster; its complexity and costs might intensify existing social inequalities, causing large groups of misfits such as people who do not fit in with the information society [1]. The elderly are always going to find it difficult to fit into new-tech easily and smoothly; they are digital immigrants learning a new language of the digital age [2], this learning process is usually laborious to them.

Learning to accommodate the existing digital social media, this can facilitate the elderly to use social media, exchange opinions and social support via the Internet. How different CMC approaches used among the elderly were being explored [3]. Indeed, the elderly can benefit from ICTs through learning to overcome their anxiety on using the computer, and then to gradually accommodate this new communication approach. Not every elderly person can go through this learning process, especially those with lower education and speaking dialect primarily.

The social media nowadays are designed by so-called digital natives and the elderly attributes about media usage and communications are almost never being taken into account. As digital social media has become an influential new media [4], though social media connects people via the Internet, this is different from previous media usage and interpersonal interaction that the elderly are familiar with. For improving the elderly interpersonal and social relationships, we have to understand the media usage and characteristics of the elderly social life.

The digital divide in the information society brings about the issues of the inequalities on using new communication approaches [5], those also faced by the elderly. Developing an adequate new media environment for the elderly can facilitate them to participate in social media. The elderly are willing to use social media to connect with people and contribute contents, such as by expressing their opinions, only if they have sufficient support on using these new media [6,7]. The objective of this research is to investigate the differences between the previous media and digital social media usage, for developing an adaptive social media which can fit the elderly's needs, close to their custom, and be more acceptable to the elderly. The social media is designed positively for the elderly so that it can support the elderly current practices and understanding rather than forcing them to adopt new and alien ways of keeping in touch [8].

\section{Literature Review}

Being old is not just a physical degradation, but also a social status that the elderly seek to manage, sometimes resisting, sometimes accepting [9]; as the attitudes to families and friendships are concerned, and the role in those relationships, the elderly seem to have their own perspectives.

The elderly have unique design requirements that are probably stronger than the potential for their experiencing physical or cognitive decline [9]. Thus, for giving the elderly the option to participate in mediated social interaction online through technology, developing the social media for the elderly should take their life situations, habits and attitudes into account [10]. For having social media used and accepted, it must not only be usable but also support current patterns of communication wherever possible and fit into the environment in which the elderly live, rather than introducing a 
completely new usage pattern to the elderly [8]. In the following sections, we first review the related researches in media usage among the elderly, and then the attitudes of the elderly on communications and relationships.

\subsection{Media Usage among the Elderly}

Those media the elderly are familiar with play the important roles in their lives; the elderly rely on watching TV which can provide information and entertainment, and the telephone as a communication media which serves them to contact people. In addition, there are researches that claimed the elderly would feel anxious on using new-tech products [11-13].

Watching TV is almost an important activity among the elderly in their daily lives; the reason they always watch TV is having no other adequate leisure activity to do; also the TV was very familiar to them when they were young. There are four reasons why the elderly are used to watching TV: 1) for relaxation, entertainment and as a companionship; 2) for getting information that can provide materials to talk; 3) watching TV can be a way to escape temporarily and give a chance to interact with friends or families by talking about the TV programs; 4) Passing time by watching TV had been formed as a habit in life [14].

The telephone is the preferred way of keeping in touch among the elderly, because it is seen as easy to use, and allows normal behavior, like chatting; and personal telephone calls are not recorded, or ever heard by another; the telephone also allows richer communication by allowing the user to hear someone's mood, or health, in their voice. Its immediacy also gives benefits by offering security in case help is needed quickly [8].

Other existing researches explored different devices that the elderly were interacting with, practically focused on the adaption of new functions and human interface $[12,15-17]$. For example, the touch-based user interface would be easy to learn and adopt among the elderly, and they would be able to successfully use it regardless of the physical or cognitive weaknesses of the elderly [12].

The problems faced by the elderly in using and engaging with interactive media are currently less associated with physical and cognitive factors, more related to their previous experiences and attitudes [18]. There are main obstacles that the elderly face in contributing content by adopting user-generated content (UGC), these are: 1) with respect to affect, personal integration, affiliation, release of tension, and creation, they did not perceive what UGC can offer them; 2) fear that the systems are too hard to learn, and concomitant low expectations towards support that can help them; 3) lack of social influences, the elderly did not perceive that someone important to them thought they should use the application; 4) pre-established negative attitudes towards digital technologies, including computer anxiety and concerns about privacy [6].

\subsection{Attitudes of the Elderly to Communications and Relationships}

Previous researches did not explore the needs of the elderly as a group suffering cognitive or physical decline, instead, focusing on the elderly attitudes to keeping in touch $[10,15]$. For example, the elderly want to feel that real contact with someone, contacts reaching a level of intimacy, and that something representing for them can be put into the communication, such as their voices or handwriting [15]; whereas 
inputting text with the computer can produce neater and consistent text that is more readable, helping the elderly lift the worry of someone else's confusion with their handwriting [13].

Concerning the time spent on relationships, the elderly are more motivated to spend time on relationships that are emotionally rewarding and of significance to them, such as their families and close friends, and are less motivated to acquire new knowledge about the social world by meeting new people [15]. In the family relationship, the assumptions about symmetry being inherent in family ties can be misleading; that asymmetry is often a better way of accounting for the attitudes of the elderly to their families; asymmetry also offers a better basis for design [9].

In terms of social media usage, the factors most hindering the elderly to use SNSs would be: 1) The elderly fear their personal information being used by malicious third parties, because not only confusion on how to adjust the SNS privacy settings, also their lack of confidence in their personal computer skills; 2) fear of accidental social blunders in mediated social interaction also due to not understanding privacy settings and privacy management; 3) incompatibility of their perceptions of social relationships with their preconceptions and assumptions about SNSs ; 4) the extension of the interaction habits formed through very-long-term relationships to a new interaction environment was regarded as costly [10]. Those concerns should be taken into account of designing the social media for the elderly.

From the elderly viewpoint there is the differentiation between the time for communication and the time not to communicate; they are careful not to intrude on others, and would like to be treated by others in the same way [15]. There are elderly people who hardly understand that communications among the younger generation using CMC are always continuous but transient. However, the elderly who can communicate by e-mail think that they can send informal messages by e-mail to breach the restriction of time zone, and also allow them to compose an e-mail at their leisure [8].

\section{Method}

A ground theory approach is applied in this research. We firstly carry out in-depth interviews of five 55-60-year-old subjects for exploring the patterns of the elderly on media usage and social connection in daily life. This semi-structured interview included several approaches of media usage investigations: 1) what they have and use? 2) how they connect with others? 3) what they do? 4) when and where they use them? And in the second part protocol analysis, we use NVivo to code the transcripts and sort them into six main categories.

\subsection{The Subjects and the Interview Circumstances}

There were five elders to be invited to become the subjects in this research. These subjects interviewed in Taiwan have the following attributes: 1) their ages are 55-60 year-old, because of our research focusing on those who are about to retire or have retired; 2) they are not familiar with using the personal computer for communication, especially inputting traditional Chinese characters by keyboard is a challenge; 3 ) they are used to using Taiwanese to communicate with people, that is a dialect pervasive in Taiwan among the elderly in this age. 
The circumstances in which the interviews were carried out are familiar to the subjects. Two subjects (one male, one female) were interviewed respectively, and three subjects (one male, two females) were interviewed as a focus group; in the context of the focus group there also were other three people whose age was under 55 year-old giving some opinions to serve to facilitate these three subjects in expressing their perspectives much more. These sessions were voice-recorded for later review and analysis.

\subsection{Questions for Interview}

The questions designed for interview are in order to realize the characteristics of media usage and social life among the elderly (Fig.1 shows the question framework); how they use media and communication devices as well as interact with their friends and relatives in daily life. Fundamentally, these questions are based on two dimensions, media usage and social life. Semi-structured interviews were carried out by asking five subjects these prepared questions and the extended questions according to the subjects' answers. After interviews voice-data was acquired and this was transcribed and analyzed in the next stage.

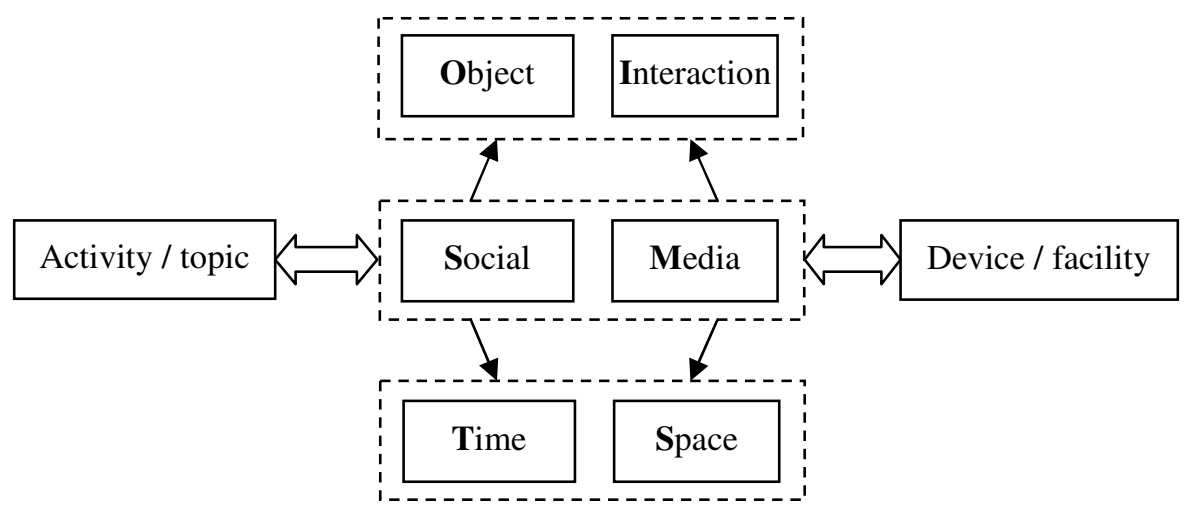

Fig. 1. The question framework: the core including two main nodes - Social and Media; Object for people contacted like friends; Interaction for the way to interact with people or media; Time for the questions related to time and Space for questions related to space

\subsection{Analysis}

In the process of analysis there are four steps: 1) convert the voice-recorded data of interviews to transcripts; 2) preliminary free coding; 3 ) organize the free codes to tree codes; 4) find the elderly patterns through examining these coded data back and forth.

Firstly all conversations in the interviews were converted to transcripts using a word processor and then imported to NVivo, the software used to code and analyze data. Secondly the transcripts were read and coded to be free codes. Thirdly the free codes were organized into tree codes, meaning to organize codes in hierarchy, some of these codes were combined and renamed, and then classified these codes under the 
six main categories according to the framework of the designed questions. These categories are: 1) social, 2) media, 3) time, 4) space, 5) object and 6) interaction. Finally these coded data were examined to find the patterns of the elderly media usage and social life.

\section{Findings}

Some trends appeared from the analysis. As the interview questions were designed for investigating the patterns of the elderly in media usage and social life, these findings are divided into two sections:

\subsection{Media Usage of the Elderly}

In the categories of media and time, the subjects expressed they spend much time watching TV, at least 3 hours a day. They always turn on the TV when having nothing else to do. The TV is their main media device in getting information and for entertainment in daily life. This result is in accordance with some previous research, they believe the elderly majorly acquire information and pass time by watching TV [14]. Our subjects usually watch TV news at first, and other programs would be tour and food programs, variety shows and drama series. Non-native language programs are less popular, because it made our subjects strenuous that watching these programs they need to read the subtitles on the TV screen.

Although watching TV news is for knowing what happened outside, they consider it annoying that the same TV news content televised is repeated many times in one day; also because of this, they would not be concerned about missing TV news that will be televised again soon. Due to their patterns of elderly daily life, the time they watch TV is usually fixed; they seldom change their living schedule for watching a TV program.

Other media such as radio, magazines or newspapers are used in some context by some of our subjects; they might listen to radio while driving, otherwise read newspapers or magazines while waiting for services at a store. Our subjects are not subscribing to newspapers or magazines. And because of presbyopia, the subjects expressed inconvenience that when reading newspapers or magazines; they need to wear reading glasses.

The subjects indicated that if not watching TV, they would not know how to pass time in daily life; while watching TV they can focus on the TV programs. If they cannot watch TV, they probably want to be accompanied. So the TV can act as a companion for the elderly who have much spare time in retirement.

In the categories of space and interaction, two subjects using a computer to get information on the Internet, they appreciated the selectivity of this new media that can help them to find the content that they are interested in, but it made their eyes feel tired easily when using the computer. One female subject expressed it would be better that the flexible selectivity of using a computer could be adapted to the TV in the living room. Compared to staring at the computer monitor, watching TV is more comfortable for them; also the living room environment is cozier and more suitable for a small group of people. 


\subsection{Communications and Relationships in the Elderly Social Life}

In the categories of social and interaction it reveals our subjects mainly use phone/mobile phone for connecting with people. Not only when using the phone/mobile phone can freely express in oral, also their contacts mainly use phone/mobile phone as their communication device. Three subjects expressed that they would use text messages in some situations; they usually use text messages to inform the person who could not answer the phone, or to inform many friends to attend an activity or a party. However, typing text messages on the mobile phone by Chinese phonetic input method in Bopomofo ${ }^{1}$ is not easy to use for them; and the subjects indicated the characters on the mobile phone buttons are too small to see clearly, they needed to wear reading glasses while typing text messages.

In the categories of interaction and object, it was found that having no interaction with friends and no way to learn friends' recent situation would cause losing contact with friends. The only way to maintain the relationships with friends are through the phone/mobile phone; but some of our subjects deemed that if they cannot meet friends regularly, their relationships would be estranged gradually. Our subjects believe touch in person via face-to-face interaction is important. However, the restriction of distance made them not able to meet each other regularly, with only one contacting the other by phone, which would result in the relationship fading away, and possibly, to disappear someday.

In the subjects' social networks the people who are relatives would be gathered together at weddings or funerals, which remain the important chances of interactions. Probably they would have not contacted one another for a long time, but due to these chances, their connections are hardly broken off; not like the connections with friends could be weakened or disappear, because of losing contact. One female subject expressed that when meeting classmates in the class reunion, she felt comfortable and hardly had a strange feeling; the relationships with classmates built in student life seems to easily be reconnected.

Although some of our subjects have an e-mail account, they rarely communicate with friends via e-mail because of their friends not using e-mail. The e-mails they received were almost always advertisement e-mails, as a result of leaving their e-mail address in the service applications such as bank services.

The subjects who use a computer to communicate are mainly using voice devices to have conversations, the experiences which are similar to using phone/mobile phone; sometimes, if having a webcam, they would use it to see each other.

\section{Discussions and Conclusion}

Our subjects prefer information in streaming media format, such as video and radio broadcasting, and non-native language programs are less popular. Watching TV and controlling it by a remote controller are very familiar for our subjects; they have no difficulties in selecting the program or channel that they want to watch. In terms of

${ }^{1}$ Bopomofo consisting of 37 characters and 4 tone marks is widely used as an educational tool and Chinese computer input method in Taiwan

(http://en.wikipedia.org/wiki/Bopomofo). 
that they still participate in call-in TV and radio programs, and turn on the TV or radio until they go to bed. For creating an experience similar to watching TV, referring to interactive TV would be valuable $[19,16]$.

People share opinions and daily lives to develop relationships. In relationship connection, people maintain and develop what they care about and are cared for. The research shows that the elderly prefer passive ways of gathering information; they seldom grasp or attempt information actively. Therefore they feel new media needs consistent attention or manipulation in strenuous circumstances. Also the elderly social connections are actually based on substantial relations already existed. As their existing substantial relations are not all fancy on new technologies, a big ratio have difficulty with special input devices and system of traditional Chinese.

Our subjects almost did not write letters, they were almost not used to expressing thoughts by writing. Oral expression is the straightforward way for communication to them, and they can express themselves in their familiar dialect, regardless of the frustrated feeling in accommodating the Chinese input method with a keyboard. Therefore, voice input cooperated with manuscript input is more acceptable to the elderly. Regarding the presentation of information in text, for the elderly the readability needs to be considered, text-to-speech or voice messages are also a feasible way.

For facilitating or encouraging the elderly to participate in social media, it would make uses of social media more acceptable to the elderly, the way of using social media much closer to their habits in daily life. The influences of Physical and cognitive decline, the attitudes of keeping in touch, and the ways to contact people, which all conditioned the elderly participation in social media in the digital age.

As most digital social media are formed and followed by the younger generation, to understand the media usage and behavior of previous generations is essential for bridging the inconsistent way of accommodating the accelerating artificial world, and improving the dignity of elderly life. In future studies researchers can invite the elderly to participate in a design process of social media, and then to produce a prototype to validate the requirements for this group of elderly in Taiwan.

\section{References}

1. Dijk, J.V.: The network society: social aspects of new media. Sage, Thousand Oaks (2006)

2. Prensky, M.: Digital natives, digital immigrants. On the Horizon 9, 1-6 (2001)

3. Xie, B.: Multimodal Computer-Mediated Communication and Social Support among Older Chinese Internet Users. Journal of Computer-Mediated Communication 13, 728-750 (2008)

4. Kaplan, A.M., Haenlein, M.: Users of the world, unite! The challenges and opportunities of Social Media. Business Horizons 53, 59-68 (2010)

5. Frissen, V.: The myth of the digital divide. In: E-merging media: Communication and the Media Economy of the Future, pp. 271-284. Springer, Heidelberg (2005)

6. Karahasanovic, A., Brandtzæg, P.B., Heim, J., Lüders, M., Vermeir, L., Pierson, J., Lievens, B., Vanattenhoven, J., Jans, G.: Co-creation and user-generated content-elderly people's user requirements. Computers in Human Behavior 25, 655-678 (2009)

7. Ryu, M., Kim, S., Lee, E.: Understanding the factors affecting online elderly user's participation in video UCC services. Computers in Human Behavior 25, 619-632 (2009) 
8. Dickinson, A., Hill, R.L.: Keeping in Touch: Talking to Older People about Computers and Communication. Educational Gerontology 33, 613-630 (2007)

9. Lindley, S.E., Harper, R., Sellen, A.: Designing for elders: exploring the complexity of relationships in later life. In: Proceedings of the 22nd British HCI Group Annual Conference on People and Computers: Culture, Creativity, Interaction, vol. 1, pp. 77-86. British Computer Society, Liverpool (2008)

10. Lehtinen, V., Näsänen, J., Sarvas, R.: A little silly and empty-headed: older adults' understandings of social networking sites. In: Proceedings of the 23rd British HCI Group Annual Conference on People and Computers: Celebrating People and Technology, pp. 45-54. British Computer Society, Cambridge (2009)

11. Collins, D.: A comparison of Web browser interactions by older users using a keyboard or a mouse as an input method (2008)

12. Häikiö, J., Wallin, A., Isomursu, M., Ailisto, H., Matinmikko, T., Huomo, T.: Touch-based user interface for elderly users. In: Proceedings of the 9th International Conference on Human Computer Interaction with Mobile Devices and Services, pp. 289-296. ACM, Singapore (2007)

13. Sayago, S., Blat, J.: About the relevance of accessibility barriers in the everyday interactions of older people with the web. In: Proceedings of the 2009 International CrossDisciplinary Conference on Web Accessibililty (W4A), pp. 104-113. ACM, Madrid (2009)

14. Koçak, A., Terkan, B.: Media use behaviours of elderly: A Uses and Gratifications Study on Television Viewing Behaviors and Motivations. GeroBilim Journal, Journal on Social and Psychological Gerontology 1, 70-86 (2009)

15. Lindley, S.E., Harper, R., Sellen, A.: Desiring to be in touch in a changing communications landscape: attitudes of older adults. In: Proceedings of the 27th International Conference on Human Factors in Computing Systems, pp. 1693-1702. ACM, Boston (2009)

16. Mitchell, V., Nicolle, C.A., Maguire, M., Boyle, H., et al.: Web-based interactive TV services for older users. Gerontechnology 6, 20-32 (2007)

17. Müller, C., Neufeldt, C., Schröer, L.: Designing a large social display for an old people's home (2010)

18. Turner, P., Turner, S., Van De Walle, G.: How older people account for their experiences with interactive technology. Behaviour \& Information Technology 26, 287-296 (2007)

19. Mitchell, K., Jones, A., Ishmael, J., Race, N.J.: Social TV: toward content navigation using social awareness. In: Proceedings of the 8th International Interactive Conference on Interactive TV \& Video, pp. 283-292. ACM, Tampere (2010) 